\title{
Promotion of physical activity in individuals with intellectual disability
}

Heidi I Stanish, PhD, (I) Georgia C Frey, PhD(2)

\section{Stanish HI, Frey GC. Promotion of physical activity in individuals with intellectual disability. Salud Publica Mex 2008;50 suppl 2:SI78-SI84.}

\begin{abstract}
This paper provides an overview of strategies that have been used to promote physical activity in individuals with intellectual disability. Several different approaches are discussed and the strengths and limitations of each are presented. Some determinants of physical activity for individuals with intellectual disability are also reported in an effort to better understand the factors that influence participation that could be targeted in future interventions. Recommendations for programming are provided.
\end{abstract}

Key words: physical activity; health promotion; intellectual disability
Stanish HI, Frey GC.

La promoción de actividad física en individuos

con discapacidad intelectual.

Salud Publica Mex 2008;50 supl 2:SI78-SI84.

\section{Resumen}

Este artículo presenta un panorama de las estrategias que se han empleado para promover la actividad física en individuos con discapacidad intelectual. Se discuten varios enfoques distintos y se presentan las fortalezas y limitaciones de cada uno. Se informa asimismo acerca de algunos de los determinantes de la actividad física para los individuos con discapacidad intelectual para contribuir a un mayor entendimiento de los factores que influyen en la participación y que podrían ser el objetivo de futuras intervenciones. Se proporcionan recomendaciones para la programación.

Palabras clave: actividad física; promoción de la salud; discapacidad intelectual

\section{Promotion of physical activity in people with intellectual disability}

A paper in this issue by Temple and Stanish clearly outlines what is known about the health benefits of physical activity as well as the physical activity habits of individuals with intellectual disability (ID). Although there is inadequate research to provide definitive conclusions about the health behaviors of individuals with ID, there is substantial evidence that fitness levels are low ${ }^{1,2}$ and it is accepted that engagement in physical activity must increase. In the United States, government agencies have specifically targeted individuals with ID in health promotion campaigns ${ }^{3}$ because they are considered a population at high risk for sedentary lifestyles and the associated health problems. There have been efforts to develop and evaluate strategies to improve fitness and increase activity levels. However, people with ID

(I) University of Massachusetts Boston, USA.

(2) Indiana University, Bloomington, USA. 
face some unique barriers to participation that must be addressed in order for physically active lifestyles to be realized in this population segment.

Unfortunately, the published research on physical activity promotion in people with ID has primarily been conducted in developed countries where resources are readily available. It is important that the findings reported in this paper be considered in that context. However, many principles of physical activity promotion are widely applicable and so long as the realities of a country's structure, traditions, and culture are considered, effective strategies can be developed.

\section{Samples of approaches to promote physical activity}

For more than 30 years researchers have conducted experimental studies to examine the effect of exercise training on fitness outcomes in children and adults with ID. As a result of this work, there is considerable evidence that training induces positive changes in aerobic fitness, ${ }^{4-7}$ muscular strength and endurance, ${ }^{8,9}$ and flexibility. ${ }^{10}$ It is important to note that individuals with Down syndrome may respond differently to exercise training. ${ }^{11,12}$ However, since studies have also shown fitness gains in this group, ${ }^{13}$ the same general principles of physical activity promotion apply to individuals with Down syndrome.

While researchers have continued to examine the effects of exercise on outcomes of health-related fitness, it is clear that individuals with ID adapt to increased levels of physical activity in much the same way as individuals without disabilities. Although a direct relationship exists between fitness levels and engagement in physical activity, health agencies worldwide continue to promote the accumulation of activity over attaining a state of fitness for the general public. ${ }^{14,15}$ The World Health Organization ${ }^{15}$ promotes 30 minutes of moderate intensity physical activity on all, or most, days of the week. Therefore, the greater need is for researchers to develop and test interventions that encourage individuals with ID to initiate and maintain physical activity. ${ }^{16,17}$ Once effective behavior change strategies are established for this segment of the population then positive health outcomes will result.

Research efforts to increase physical activity by individuals with ID have produced relatively consistent and positive results. While many interventions have been short-term and some have methodological shortcomings (e.g. small sample size), it is beneficial to review the findings of previous work to guide future research and practice. Tomporowski and Jameson ${ }^{18}$ paired adults with ID and nondisabled exercise partners over an 18-week walk/jog program. Partners assisted with pacing and provided ongoing verbal encouragement to motivate the participants with ID while they were engaging in activity. The training program was effective for promoting exercise behavior in that participants progressively increased their distance walked/jogged and their speed of walking/jogging over the course of the intervention. A similar approach was taken by Lavay and McKenzie ${ }^{19}$ who reported that five men with ID actively participated in a supervised walk/jog program three days per week for 12 weeks. Aerobic fitness levels increased significantly as a result of participation. Most importantly, authors noted that once the training program was discontinued, the men continued to walk/jog three days per week for a year. These studies provide evidence that, with some supervision and encouragement, adults with ID will actively engage in short-term walking programs. Walking is the most commonly reported physical activity by individuals with $\mathrm{ID}^{20-22}$ and is a cost-effective, convenient, and appropriate activity to promote for this group.

In an effort to motivate three adult women with ID to progressively increase their cycling duration on a stationary bicycle, Bennett and colleagues ${ }^{23}$ employed a token economy system of reinforcement. The token economy involved participants exchanging tokens for rewards such as outings, food, or other tangible items when exercise goals (i.e. cycling duration) were reached. Participants did alter their behavior by increasing cycling time over the course of the intervention. Similar work was conducted by Owlia, French, Ben-Ezra, and Silliman ${ }^{24}$ who used music and music videos to increase the time on task of five adolescents with profound ID who also cycled on stationary bikes. Cycling time increased in all but one participant. Todd and Reid ${ }^{25}$ used television and verbal praise to effectively increase the number of revolutions pedaled (i.e. speed of cycling) in adults with ID. The findings of these studies further indicate that individuals with ID will participate in physical activity and that level of engagement increases with positive extrinsic reinforcement.

The effectiveness of a 12-week (three days/week) low-impact aerobic dance program for improving cardiovascular endurance in adults with ID was examined. ${ }^{26} \mathrm{~A}$ second goal was to determine whether aerobic dance is motivating to individuals with ID and if participants would continue to voluntarily exercise at their worksite once the intervention was complete. A token economy system and verbal reinforcement were used to motivate attendance and participation. Overall, aerobic fitness improved as a result of engaging in aerobic dance, attendance was high, and no individuals dropped out. However, no participants engaged in 
any form of self-initiated exercise once the program was discontinued. Personalized aerobic dance videos were examined for promoting physical activity at an employment center for adults with ID. ${ }^{27}$ Approximately $80 \%$ of all employees chose to participate in the aerobic dance sessions over 10 weeks and actively engaged in moderate intensity activity for most of each 15-17 minute session. Weekly probes in the four weeks after the intervention were conducted to determine if the participants continued to engage in physical activity after the intervention was completed. A core group of individuals continued to do aerobics with the videos for a month but the level of active engagement was slightly reduced. Authors of both studies supported that adults with ID will be active if provided enjoyable opportunities like aerobics, but the direct involvement of others (i.e. staff, families) is needed to facilitate participation and promote adherence.

Pitetti and $\operatorname{Tan}^{6}$ examined participation and adherence to a 16-week worksite stationary cycling program for adults with ID. The intervention was unique because participants were trained on the cycle and no extrinsic reinforcement or prompting was involved which is reflective of an inclusive community-based activity environment. Aerobic fitness levels increased as a result of participation and adherence to the program was high with only 1 of 14 individuals dropping out for lack of interest. Authors reported, however, that once the intervention was complete participants did not voluntarily continue to exercise even though cycles remained at the worksite. Podgorski and colleagues ${ }^{28}$ investigated the effect of a 12-week physical activity intervention on the physical function of older adults with ID. Authors also evaluated whether participants would choose to engage in activity sessions and whether staff would sustain the program following the intervention. The intervention was designed in accordance with guidelines put forth by health organizations in the United States and considered variables such as space, cost, cognitive levels and communication abilities of participants, and medical issues. The physical activity sessions were conducted four days per week at a day habilitation site using direct care staff to assist. Overall, participants did improve their physical function in at least one domain (strength, flexibility, mobility and gait). Most importantly, the attendance rate for the 12 -week program was $75 \%$ and 12 of 15 participants were still active 1 year after the intervention period. In fact, staff eventually increased the number of exercise groups in order to accommodate all of the individuals who were interested in participating.

With few exceptions, past attempts to increase physical activity in individuals with ID have focused on very specific exercise goals (i.e. cycling time; walking speed) in short term programs. While the identification of effective strategies to change behavior on a small scale is both important and useful, efforts to increase healthpromoting physical activity over the lifespan need to be much broader in scope. Further, more sophisticated measures of physical activity should be employed in order to more clearly define the influence of participation on health outcomes, not fitness scores. Recently, health promotion programs have been created to target individuals with ID in an effort to change behavior and address the high prevalence of obesity. These efforts typically include physical activity as one component of healthy behavior and have aimed to provide the training and knowledge necessary to improve habits.

Marshall, McConkey, and Moore ${ }^{29}$ conducted a series of nurse-led health promotion sessions over 6-8 weeks for 25 adults with ID. Poor results on health screenings for this population prompted the intervention which was adapted from an existing program (Activate) created by the Health Promotion Agency in Northern Ireland. The content of the 2-hour sessions included information on healthy eating and exercise and the goal was to reduce body weight in overweight and obese adults. The mean weight of the group was significantly reduced and body mass index improved as a result of the classes. The authors argued that education is needed as well as suitable opportunities for physical activity and healthy eating to promote and sustain weight loss in this population.

Two recent papers report on the effectiveness of a 12-week exercise and health promotion program for adults with Down syndrome. ${ }^{13,30}$ The exercise training component of program involved sessions of cardiovascular and strength exercise three days per week for 45 minutes. Participants were taught how to use the exercise equipment safely and were supervised in groups of seven or eight by an exercise physiologist and two assistants. Heart rate monitors were used to ensure that participants trained within a target heart rate zone. The program was effective for improving cardiovascular fitness and muscular strength and small reductions in body weight were also reported. ${ }^{13}$ The health education component used the Exercise and Nutrition Health Education Curriculum for Adults with Developmental Disabilities $^{31}$ which was designed to increase understanding of health promoting behaviors, self-efficacy in performing exercise, and assist with developing health promotion goals and action planning. In addition to the documented changes in fitness, participants experienced improved psychosocial outcomes including exercise self-efficacy, life satisfaction, expected outcomes, and reported fewer cognitive-emotional barriers. ${ }^{32}$ The combination of exercise training and health education 
is unique to this curriculum and the published findings provide strong support for this approach.

Mann et al..$^{33}$ evaluated the effectiveness of a health promotion program for changing behavior and increasing knowledge in adults with ID who were overweight or obese. Further, the authors were interested in determining the interactions among diet, exercise, and knowledge and their relative contributions to changes in body mass index. The health promotion curriculum (Steps to Your Health) involved eight weekly classes that provided information on weight loss, exercise, nutritional choices, and stress reduction. An optional brisk walk with the instructor followed each 90 minute session. Participants also received two home visits to establish an individualized exercise program and a healthy diet plan. Overall, the program resulted in increased knowledge, healthier diet, more frequent exercise and a reduction in body mass index. Knowledge about a healthy lifestyle was strongly related to body mass index and weight loss. Although only frequency of physical activity was monitored in the study, it is at least an indication that changes in physical activity can occur if education on healthy behavior is provided.

The majority of studies on physical activity promotion in people with ID have been conducted in developed countries (e.g. United States, United Kingdom) where resources are available to support this segment of the population. Some of the interventions used residential and day program staff to administer and support the program. Others involved community facilities, employment centers, and residential settings where equipment was accessible and trained supervisors could facilitate efforts. In order to develop, implement, and evaluate effective strategies for behavior change in people with ID, it is important that researchers and service providers consider the realities of their own culture as well as the available resources. Since there seems to be no published literature on physical activity promotion for adults with ID in economically disadvantaged countries, it is difficult to determine which approaches are most generalizable. However, many of the physical activity promotion principles reviewed in the previous work have the potential to be applied in developing countries.

\section{Determinants of participation in physical activity}

The identification of factors that influence participation in physical activity by individuals with ID is of the utmost importance if health promotion efforts are to be effective. While the determinants of physical activity as well as sedentary behavior have been studied exten- sively in children and adults without disabilities, there is limited published information on the facilitators and barriers to participation in people with ID. The scant research in this area can likely be attributed the contention that physical activity behavior is complex and is influenced by numerous factors in various domains. Gathering valid data on such multifaceted variables could prove challenging in people with ID. However, some researchers have used qualitative methodology in attempts to describe why individuals with ID display low levels of physical activity.

Messent, Cooke and Long ${ }^{34}$ conducted interviews with residential staff, day program staff, and adults with ID to examine the barriers that exist to physically active lifestyles. The staff groups reported that their ability to provide physical activity opportunities to the individuals with ID that they serve was limited by many variables. The barriers noted were 1) limited options and choices for leisure in the community for people with ID, 2) limited financial resources required for services like transportation and staff, 3) staffing ratios that precluded the adults with ID from having the support that they required to engage in an activity, 4) limited financial resources of people with ID required for program/facility fees and transportation, and 5) unclear policy guidelines for residential and day program service provision. The individuals with ID expressed an interest in participating in physical activities and also reportedly enjoyed participating in a 10 -week program administered by the authors. While the interpretation of the interviews was somewhat difficult, it was apparent that people with ID had little control over their environment and were provided few opportunities to be active.

The expected outcomes of exercise and the socioeconomic and access barriers to exercise were examined in adults with Down syndrome and their caregivers using surveys. ${ }^{32}$ The aim of the study was to identify determinants of exercise behavior to better inform behavior change strategies. Lack of energy, too difficult, and boring were the most commonly reported cognitive-emotional barriers by people with Down syndrome while caregivers perceived that lack of interest, lack of energy, and too lazy to be the most dominant barriers. In regards to access, the main barriers reported by individuals with Down syndrome were no transportation, costs too much, and no one to show how to exercise. Caregivers agreed that transportation and cost were the significant barriers to access. Results indicated that the best predictors of physical activity participation are age of the person with Down syndrome, outcome expectations of the caregiver regarding the benefits of exercise, and access barriers. It is clear from this work, as well as previous studies, that health education is needed for 
people with ID and their caregivers and that the barriers to access must be addressed.

Frey, Buchanan, and Rosser Sand $\mathrm{t}^{35}$ used multiple data sources including accelerometry, in-depth interviews, and observation in order to describe and understand the physical activity behavior of adults with ID. The participants perceived several barriers to participation in physical activity including concerns about job/ life, cost of activities, weather, time, transportation, and health issues. Many of the perceived barriers were supported by caregivers. Additionally, there was a consensus among people with ID and their caregivers that more guidance was required for people to engage in activity. People generally felt that outside assistance such as instructors and specialized programs and facilities was needed in order for adults with ID to be active. It is of concern that sedentary behavior was actually reinforced in people with ID by the individuals most responsible for supporting their lives. In some cases, coaches, doctors, and caregivers actually discouraged physical activity because they had concerns over safety and health. Despite spending their leisure time predominantly inactive, adults with ID did feel that physical activity had benefits such as rewards (e.g. medals at Special Olympics), looking good, social factors, and feeling good. Shapiro' $\mathrm{s}^{36}$ work with Special Olympians also identified winning ribbons and medals as motive for involvement as well as playing with other people, getting exercise, and fun.

An article by Lotan, Henderson, and Merrick ${ }^{37}$ provided insight into barriers to physical activity participation by adolescents with ID as well as strategies for programming. Many physical and social barriers were noted including: lack of trained personnel to work with this group, overprotection by medical professionals, lack of supervision, inadequate facilities, physiological impairments, exclusion, and others. It appears that the factors that negatively influence participation in physical activity are similar in adolescence as adulthood.

A number of determinants of physical activity participation in people with ID appear to be similar to those identified in general population. However, certain barriers like cost of services and transportation may be further exaggerated for people with disabilities who often have limited finances and independence. Also, since children and adults with ID often rely on caregivers for support, they face unique barriers related to supervision that are not as relevant in nondisabled groups. It is concerning that, in some cases, the individuals mostly responsible for the health and wellness of people with ID may actually discourage physical activity. It is clear that physical activity promotion for people with ID must take into account the barriers faced by this population and barrier-free opportunities should be developed.

\section{Recommendations for physical activity promotion}

Based on the literature reviewed in this paper, there is evidence that individuals with ID will engage in, and adhere to, meaningful physical activity if given the opportunity to do so. While more work is warranted, the research base is encouraging. It is thought that individuals with ID are not motivated to seek out opportunities to be physically active, therefore, reinforcement strategies and high supervision have typically been used in efforts to promote activity. It is true that family members, residential care providers, employers, and/or other individuals that support people with ID will play a role in identifying opportunities to engage in activity and assist with participation. Therefore, an important step toward improving health behaviors is ensuring that care providers have the knowledge, skills, and resources to facilitate healthy living.

There are a number of health promotion curriculums being developed to teach individuals with ID about the importance of physical activity and other health behaviors. ${ }^{29,31}$ Although information alone may not be adequate to stimulate behavior change, it is critical that people with ID perceive the benefits of physical activity. ${ }^{33}$ Educational plans should accompany direct activity programs so that people have both the knowledge and the skills to pursue active lifestyles. Even more importantly, physical activity promotion must involve care providers. If care providers are not aware of the benefits of physical activity and do not have the skills to assist people with ID then it is unlikely that efforts will be sustainable.

Since people with ID may not have the skills or understanding to exercise safely, they may perceive it as too difficult. ${ }^{32}$ Therefore, training is required for professionals who provide physical activity opportunities in the community. Fitness professionals are often unable to include people with ID in existing programs because they lack the knowledge required to modify activities to accommodate all ability levels. As a result, lack of trained professionals ${ }^{37}$ and too few opportunities are common barriers to participation. ${ }^{34}$ Service providers need to be trained on safety, activity modification, instructional strategies, inclusion, and communication if they are to successfully promote participation by people with ID.

The barriers of transportation, cost of service, and lack of needed supervision/assistance are ongoing 
and are somewhat challenging to address. However, physical activity opportunities do not have to be expensive and can include home- or work-based programs where transportation is not an issue. In addition, specialized equipment is not necessary since exercise videos, aerobic dance, cycling, and walking are all excellent activities for people with ID. Including physical activity into the daily routines of people with ID can also promote health. For example, increasing walking intensity (i.e. speed) to and from work was proposed as a strategy to increase appropriate physical activity.2 Reducing sedentary time, like television watching, is also essential to health promoting physical activity. Staff and family members can facilitate activity opportunities ${ }^{28}$ to reduce the need for paid instructors and high levels of supervision in the community.

Many suggestions have been put forth by researchers and practitioners to encourage people with ID to engage in physical activity. The following strategies are examples that could be considered when implementing programs for people with ID:

- Include motivational strategies and positive reinforcement.

- Include low to moderate intensity activities such as walking.

- Ensure that the activity is fun and involves social interaction.

- Involve participants in activity selection and decision making.

- Select activities that are age-appropriate.

- Conduct programs in community-based environments where there is opportunity for inclusion (but consider participant preferences).

- Be prepared to modify activities to accommodate all abilities.

- Monitor progress and set activity goals.

Lotan et al. ${ }^{37}$ suggested in their review specific strategies for adolescents with ID including: parents and teachers setting an example and acting as role models; considering adolescents preferences and making activities enjoyable; using peers and friends in activity opportunities; combining efforts of families, health professionals; and community organizations to promote active living; and using a balanced program involving endurance, flexibility, and strength training.

Ideally, direct programs would be tied to larger health promotion efforts that address nutrition and other health behaviors like reduction of tobacco use and stress reduction. The multitude of barriers and facilitators that influence accessibility must be addressed.

\section{Conclusions}

It is highly encouraging that the National Institute of Public Health of Mexico has identified the need to address the health disparities among individuals with ID. By publishing a thematic issue that specifically addresses the salient health concerns of people with ID, the Institute has made healthy living a priority for a traditionally underserved population. While large scale efforts are needed to affect health policy and stimulate widespread changes in behavior, it may be more effective to implement community-based programs and local campaigns when starting to address the problem of physical inactivity. As indicated in this review, early attempts to increase physical activity by people with ID involved small scale, exploratory studies. As health for people with ID has evolved into a greater priority in developed countries like the United States, the physical activity promotion activities have grown to have a greater impact. Mexico can gain insight into the process of physical activity promotion for people with ID from the strengths and limitations of work conducted around the world which positions them well for success.

\section{References}

I. Fernhall B, Pitetti KH. Limitations to physical work capacity in individuals with mental retardation. Clin Exerc Physiol 200।;3:176-185. 2. Graham $A$, Reid $G$. Physical fitness of adults with an intellectual disability:A |3-year follow-up study. Res Q Exerc Sport 2000;7|:|52-|61. 3. U.S. Public Health Service. Closing the gap:A national blueprint for improving the health of individuals with mental retardation. USDHHS 2002. 4. Frey GC, McCubbin JA, Kasser S, Skaggs S. Physical fitness of trained runners with and without mental retardation. Adapt Phys Activ $Q$ 1999;16:126-137.

5. Lotan M, Isakov E, Kessel S, Merrick J. Physical fitness and functional ability of children with intellectual disability: Effects of a short-term daily treadmill intervention. Scientific World Journal 2004;14:449-457.

6. Pitetti KH,Tan DM. Effects of a minimally supervised exercise program for mentally retarded adults. Med Sci Sports Exerc 1991;23: 594-60I.

7. Pommering, TL et al.Effects of an aerobic exercise program on community-based adults with mental retardation. Ment Retard 1994;32:218-226.

8. Rimmer JH, Kelly LE. Effects of a resistance training program on adults with mental retardation. Adapt Phys Activ Q 1991;8: I46-153.

9. Suomi R, Surburg PR, Lecius P. Effects of hydraulic resistance strength training on isokinetic measures of leg strength in men and women with mental retardation. Adapt Phys Activ Q 1995; 12:377-387.

10. Stopka C, Morely K, Siders R, Schuett J, Houck A, Gilmet Y. Stretching techniques to improve flexibility in Special Olympics athletes and their coaches.J Sport Rehabil 2002; I :22-34.

II. Millar A, Fernhall B, Burkett L. Effects of aerobic training in adolescents with Down syndrome. Med Sci Sports Exerc 1993;25:309-315. 
12.Varela A, Sardinha LB, Pitetti KH. Effects of an aerobic rowing training regimen in young adults with Down syndrome. Am J Ment Retard 200I;106:135-I 44

13. Rimmer JH, Heller T,Wang E,Valerio I. Improvements in physical fitness in adults with Down syndrome. Am J Ment Retard 2004; 109:165- 174. I4. U.S. Department of Health and Human Services. Healthy People 2010: Understanding and improving health. (2nd ed.). USDHHS 2000. 15.World Health Organization. WHO global strategy on diet, physical activity and health. [Online]. 2003 [cited 2006 Dec 12].Available from: URL: http://www.who.int/dietphysicalactivity/strategy/eb I 344/en/index.html. 16. Rimmer JH, Braddock D. Health promotion for people with physical, cognitive, and sensory disabilities:An emerging national priority. Am J Health Promot 2002;16:220-224.

17. Rimmer JH, Braddock D, Pitetti KH. Research on physical activity and disability:An emerging national priority. Med Sci Sports Exerc 1996;28: I366-1372.

18. Tomporowski PD, Jameson LD. Effects of a physical fitness training program on the exercise behavior of institutionalized mentally retarded adults. Adapt Phys Activ Q 1985;2:197-205.

19. Lavay B, McKenize TL. Development and evaluation of a systematic walk/run program for men with mental retardation. Educ Train Ment Retard 199|;September:333-34I.

20. Draheim CC, Williams DP, McCubbin JA. Prevalence of physical inactivity and recommended physical activity in community-based adults with mental retardation. Ment Retard 2002;40:436-444.

2I. Stanish HI, Draheim CC.Assessment of walking activity using a pedometer and survey in adults with mental retardation. Adapt Phys Activ Q 2005;22:136-145.

22. Temple VA, Anderson C,Walkley JW. Physical activity levels of individuals living in a group home. J Intellect Dev Disabil 2000;25:327-34I. 23. Bennet F, Eisenman P, French R, Henderson H, Shultz B. The effect of a token economy on the exercise behavior of individuals with Down syndrome. Adapt Phys Activ Q 1989;6:230-246.

24. Owlia G, French R, Ben-Ezra V, Silliman LM. Influences of reinforcers on the time-on-task performance of adults who are profoundly mentally retarded. Adapt Phys Activ Q 1995; 12:275-288.

25. Todd T, Reid G. Television and verbal encouragement as exercise reinforcers for persons with severe mental handicaps. Palaestra 1992; summer:42-47.
26. Cluphf D, O'Connor J, Vanin S. Effects of aerobic dance on the cardiovascular endurance of adults with intellectual disabilities. Adapt Phys Activ Q 200I;|8:60-7I.

27. Stanish HI, McCubbin JA, Draheim CC, van der Mars H. Participation of adults with mental retardation in a video- and leader-directed aerobic dance program. Adapt Phys Activ Q 200 I; 18: I42-I55.

28. Podgorski C, Kessler K, Cacia B, Peterson DR, Henderson CM. Physical activity intervention for older adults with intellectual disability: Report on a pilot project. Ment Retard 2004;42:272-283.

29. Marshall D, McConkey R, Moore G. Obesity in people with intellectual disabilities: the impact of nurse-led health screenings and health promotion activities. JAdv Nurs 2003;4I:147-153.

30. Heller T, Hsieh K, Rimmer J. Barriers and supports for exercise participation among adults with Down syndrome.J Gerontol Soc Work 2002;38:16I- 178.

3I. Heller T, Marks BA, Ailey SH. Exercise and Nutrition Health Education Curriculum for Adults with Developmental Disabilities. Chicago: University of Illinois at Chicago, Rehabilitation Research and Training Center on Aging and Developmental Disabilities. Department of Disability and Human Development 200I.

32. Heller T, Hsieh K, Rimmer JH. Attitudinal and psychosocial outcomes of a fitness and health education program on adults with Down syndrome. Am J Ment Retard 2004; 109:175-185.

33. Mann J, Zhou H, McDermott, Poston M. Healthy behavior change of adults with mental retardation: Attendance in a health promotion program. Am J Ment Retard 2006; I I :62-73.

34. Messent PR, Cooke CB, Long J. Physical activity, exercise and health of adults with mild and moderate learning disabilities. $\mathrm{Br} J$ Learn Disabil 1998;26:17-22.

35. Frey GC, Buchanan A, Rosser Sandt DD. “'’d rather watch TV”: An examination of physical activity in adults with mental retardation. Ment Retard 2005;43:24I-254.

36. Shapiro DR. Participation motives of Special Olympics athletes. Adapt Phys Activ Q 2003;20:150-165.

37. Lotan M, Henderson CM, Merrick J. Physical activity for adolescents with intellectual disability. Minerva Pediatr 2006;58,219-226. 